\title{
Acute effect of daily fine particulate matter pollution on cerebrovascular mortality in Shanghai, China: a population-based time series study
}

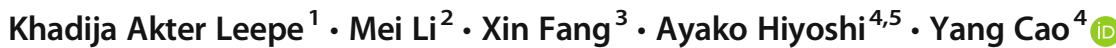

Received: 7 March 2019 / Accepted: 4 June 2019 /Published online: 1 July 2019

(C) The Author(s) 2019

\begin{abstract}
Numerous studies have investigated the impacts of ambient fine particulate matter $\left(\mathrm{PM}_{2.5}\right)$ on human health. In this study, we examined the association of daily $\mathrm{PM}_{2.5}$ concentrations with the number of deaths for the cerebrovascular disease on the same day, using the generalized additive model (GAM) controlling for temporal trend and meteorological variables. We used the data between 2012 and 2014 from Shanghai, China, where the adverse health effects of $\mathrm{PM}_{2.5}$ have been of particular concern. Three different approaches (principal component analysis, shrinkage smoothers, and the least absolute shrinkage and selection operator regularization) were used in GAM to handle multicollinear meteorological variables. Our results indicate that the average daily concentration of $\mathrm{PM}_{2.5}$ in Shanghai was high, $55 \mu \mathrm{g} / \mathrm{m}^{3}$, with an average daily death for cerebrovascular disease (CVD) of 62 . There was $1.7 \%$ raised cerebrovascular disease deaths per $10 \mu \mathrm{g} / \mathrm{m}^{3}$ increase in $\mathrm{PM}_{2.5}$ concentration in the unadjusted model. However, $\mathrm{PM}_{2.5}$ concentration was no longer associated with CVD deaths after controlling for meteorological variables. The results were consistent in the three modelling techniques that we used. As a large number of people are exposed to air pollution, further investigation with longer time period including individual-level information is needed to examine the association.
\end{abstract}

Keywords Fine particulate matter $\cdot$ Cerebrovascular mortality $\cdot$ Generalized additive model $\cdot$ Multicollinearity $\cdot$ Principal component analysis $\cdot$ Shrinkage smoother $\cdot$ Least absolute shrinkage and selection operator

\section{Introduction}

Air pollution represents the biggest environmental risk to health, and outdoor air pollution alone has been associated with an estimated three million premature deaths in a recent year (World Health Organization (WHO), 2016). Harmful

Responsible editor: Philippe Garrigues

Yang Cao

yang.cao@oru.se

Khadija Akter Leepe

khadija.akter.leepe@gmail.com

Mei Li

mei.li@ regionorebrolan.se

Xin Fang

fx63783985@hotmail.com

Ayako Hiyoshi

ayako.hiyoshi@oru.se substances, such as carbon monoxide, nitrogen dioxide, and heavy metals from natural and unnatural origins, including volcanoes to industrial activities, vehicle emission, and combustion of fossil fuel, can take the forms of solid particles, liquid droplets, or gasses (Fiordelisi et al. 2017). Fine particulate matter with a size less than or equal to $2.5 \mu \mathrm{m}$ in

1 Department of Applied Statistics, School of Business, Örebro University, Örebro, Sweden

2 Center for Assessment of Medical Technology in Örebro, Örebro University Hospital, Örebro, Sweden

3 Unit of Biostatistics, Institute of Environmental Medicine, Karolinska Institutet, Solna, Sweden

4 Clinical Epidemiology and Biostatistics, School of Medical Sciences, Örebro University, Örebro, Sweden

5 Department of Public Health Sciences, Stockholm University, Stockholm, Sweden 
diameters $\left(\mathrm{PM}_{2.5}\right)$ is the sum of such solid or liquid pollutants suspended in the air and particularly important as it tends to stay longer in the air because of its small size (Lin et al. 2018), increasing the chance of inhalation.

$\mathrm{PM}_{2.5}$ has been shown to have significant adverse effects on human health, including reduced lung function and increased risk of chronic bronchitis, heart disease, lung cancer, and various forms of cardiovascular and cerebrovascular diseases and mortality (Fiordelisi et al. 2017; Lu et al. 2015). Mechanisms linking $\mathrm{PM}_{2.5}$ and human health outcomes have not been fully understood, but unlike larger particles, small particles like $\mathrm{PM}_{2.5}$ can penetrate deep into the respiratory system or absorbed into the bloodstream and may increase systemic inflammation and alter autonomic nervous system activity (Fiordelisi et al. 2017; Shah et al. 2015).

Although air quality has been improved over the years, many cities and regions in European and Asian countries still suffer from $\mathrm{PM}_{2.5}$ concentration higher than $10 \mu \mathrm{g} / \mathrm{m}^{3}$ on an annual average, above the limit recommended by the WHO (Thunis et al. 2017). An increase in $\mathrm{PM}_{2.5}$ concentration by 10 $\mu \mathrm{g} / \mathrm{m}^{3}$ was associated with $8-27 \%$ higher lung cancer risk (Pope et al. 2002; Turner et al. 2011) and 4\% higher allcause mortality risk (Pope et al. 2002), while a reduction by $10 \mu \mathrm{g} / \mathrm{m}^{3}$ expands life expectancy for 0.35 years (Correia et al. 2013). Emergency hospital admissions for cerebrovascular disease increased by $1.3 \%$ for every $\geq 10 \mu \mathrm{g} / \mathrm{m}^{3}$ increase in $\mathrm{PM}_{2.5}$ concentration (Leiva et al. 2013).

The aim of our study was to evaluate the acute effect of $\mathrm{PM}_{2.5}$ pollution on cerebrovascular disease (CVD) mortality using data from Shanghai, China, between 2012 and 2014. We focused on the influence of $\mathrm{PM}_{2.5}$ concentration on death during the same day. As we adjusted for a number of meteorological variables and some of them show multicollinearity, the secondary aim was to examine the impact of the difference of adjustment techniques that are commonly used in the presence of collinearity: principal component analysis (PCA), shrinkage smoother, the least absolute shrinkage and selection operator (LASSO) methods.

\section{Materials and methods}

\section{Study setting and data}

The data used this study include daily observations on the number of CVD deaths, $\mathrm{PM}_{2.5}$ concentrations, and meteorological conditions between 2012 and 2014 in Shanghai, China, with a total of 1091 observed days. During the study period, the population of Shanghai was approximately 24.0 million, and the life expectancy was around 83 years (Shanghai Statistics Bureau 2014). The detailed description of the data was published elsewhere (Fang et al. 2017). Briefly, daily average $\mathrm{PM}_{2.5}$ concentrations between January
1, 2012, and December 31, 2014, were obtained from the air quality monitoring station of the US Consulate General in Shanghai and the Shanghai Meteorological Bureau. Only the measurements from a single air quality monitor station were available during the study period. The daily mortality data during the corresponding time period for all the 16 administrative districts in Shanghai were obtained from the Causes of Death Registry of Shanghai Municipal Centre for Disease Control and Prevention. The causes of death were coded according to the International Disease Classification Codes, version 10 , and the codes for cerebrovascular diseases deaths were I60-I69. Thirteen meteorological variables and the day of the week were used as potential confounding factors.

\section{Statistical model}

Mean, standard deviation (SD), median, the first quartile (Q1), and the third quartile (Q3) were calculated for daily CVD deaths, $\mathrm{PM}_{2.5}$ concentrations, and meteorological variables. Univariate associations between CVD mortality and $\mathrm{PM}_{2.5}$ and meteorological variables were evaluated using a generalized linear model with a log link function. Generalized additive model (GAM) was used for multivariate analysis. The GAM is a generalization of the generalized linear model and is widely used in time series studies on health effects of air pollution because it does not expect a particular functional form of a relationship, and is flexible for modelling nonlinear associations (Crawley 2013). It exploits the quantity of a response variable $Y$ from a given distribution with different independent variables by estimating non-parametric functions of the independent variables, which are connected to the response variable through a link function. Because of the nonlinear relationship between death counts and weather conditions, and exponential form of daily death counts within a fixed period of time, the effect of $\mathrm{PM}_{2.5}$ on daily deaths in the present study was modelled as:

$\log [E(Y)]=\log \left[\mu_{t}\right]=\beta_{0}+\beta_{1} X_{t}+\sum_{i=1}^{n} f_{i}\left(Z_{i}\right)+\beta_{j} D_{j}$

and $Y \sim$ Poisson $\left(\mu_{t}\right)$

where $E(Y)$ is the expected mean of deaths on day $t, \beta_{0}$ and $\beta_{1}$ are the intercept and slope term respectively, $X_{t}$ is the $\mathrm{PM}_{2.5}$ concentration on a day $t, f$ is the smoothing function, $Z_{i}$ is the confounding variables (i.e., time and weather conditions), and $D_{j}$ is the dummy variables of the day of the week (DOW) and $j=1,2, \ldots, 6$.

The smoothing functions $f_{i}$ are composed of nonparametric splines of confounding variables. Splines are polynomial curves that are connected at inner knots. The widely used splines in GAM are defined as the natural spline, cubic spline, or B-spline with a pre-specified number of knots. In our study, two types of smoothing splines, cubic regression spline ( $c r)$ and thin plate spline ( $t p)$, were used and compared. 
To avoid over-fitting, for each of confounding variables of time and weather conditions, the optimal number of knots (k) was selected based on Akaike's information criterion values using iterative processes. We compared $k=4,5,6,7$, and 8 because most of the studies show that $k=3$ is sufficient, and often there was no notable improvement with more than 8 knots.

The coefficient of $\mathrm{PM}_{2.5}$ multiplying by 10 is interpreted as a percentage increase in cerebrovascular deaths per $10 \mu \mathrm{g} / \mathrm{m}^{3}$ increment in $\mathrm{PM}_{2.5}$ concentration.

\section{Controlling for multicollinearity}

Except for controlling for temporal trends in daily cerebrovascular deaths, meteorological variables were considered as potential confounding factors (Jimenez-Conde et al. 2008). Multicollinearity among 13 meteorological variables was examined using the Spearman correlation and variance inflation factor (VIF), and statistically significant multicollinearity was found among the variables. To deal with the multicollinearity and high dimensionality of these confounding variables, we used three different approaches. The first approach used the principal component analysis (PCA) method to convert the variables into mutually orthogonal principal components (PCs) (Yang et al. 2015). Because the reduction of the number of variables, or dimensionality, on the other hand, may lead to a loss of useful information about the original data, we also repeated the analysis using shrinkage smoothers as an alternative approach. Shrinkage is the procedure of compressing extreme values towards a central value (Tibshirani 1996). The benefit of using the shrinkage smoothers is to shrink less important variables into zero (Marra and Wood 2011). When using PCA and shrinkage techniques, we applied both $\mathrm{cr}$ and tp splines. The third approach used the least absolute shrinkage and selection operator (LASSO) regularization, which selected variables using a penalty $\alpha=1$ (Friedman et al. 2010).

All analyses were conducted in $\mathrm{R}$ version 3.5.1 ( $\mathrm{R}$ Foundation for Statistical Computing, Vienna, Austria) using packages $p s y c h, m g c v$, and glmnet. Two-sided $p$ values less than 0.05 are considered statistically significant.

\section{Results}

\section{Descriptive statistics and univariate association}

The descriptive statistics of daily mortality caused by cerebrovascular diseases, $\mathrm{PM}_{2.5}$ concentrations, and the meteorological variables are presented in Table 1. The mean of daily $\mathrm{PM}_{2.5}$ concentration is $55 \mu \mathrm{g} / \mathrm{m}^{3}$. The daily average deaths for the cerebrovascular diseases are 62 over the study period. The estimated coefficient with $\mathrm{PM}_{2.5}$ is $1.653 \times 10^{-3}$, corresponding to a $1.653 \%$ increment in daily CVD deaths per 10

Table 1 Descriptive statistics and univariate association for the risk of cerebrovascular disease death

\begin{tabular}{|c|c|c|c|c|c|c|c|c|}
\hline Variable & Mean & $\mathrm{SD}$ & Median & Q1 & Q3 & $\beta$ & SE & $p$ value \\
\hline CVD deaths & 62 & 14 & 60 & 51 & 71 & & & \\
\hline $\mathrm{PM}_{2.5}\left(\mu \mathrm{g} / \mathrm{m}^{3}\right)$ & 55 & 39 & 46 & 29 & 69 & $1.653 \times 10^{-3}$ & $9.219 \times 10^{-5}$ & $<0.001$ \\
\hline PreAvg (kPa) & 101.61 & 0.88 & 101.51 & 100.82 & 102.26 & 0.1576 & 0.0045 & $<0.001$ \\
\hline PreMax (kPa) & 101.80 & 0.90 & 101.84 & 101.01 & 102.49 & 0.1586 & 0.044 & $<0.001$ \\
\hline PreMin $(\mathrm{kPa})$ & 101.34 & 0.87 & 101.35 & 100.58 & 103.67 & 0.1531 & 0.0046 & $<0.001$ \\
\hline TemAvg $\left({ }^{\circ} \mathrm{C}\right)$ & 17 & 9 & 18 & 9 & 24 & -0.0172 & 0.0004 & $<0.001$ \\
\hline $\operatorname{TemMax}\left({ }^{\circ} \mathrm{C}\right)$ & 21 & 9 & 22 & 13 & 28 & -0.0160 & 0.0004 & $<0.001$ \\
\hline TemMin $\left({ }^{\circ} \mathrm{C}\right)$ & 14 & 9 & 15 & 6 & 22 & -0.0168 & 0.0004 & $<0.001$ \\
\hline HumAvg (\%) & 70 & 13 & 72 & 62 & 79 & -0.0011 & 0.0003 & $<0.001$ \\
\hline HumMin (\%) & 51 & 18 & 51 & 38 & 63 & -0.0011 & 0.0002 & $<0.001$ \\
\hline RainVol (mm) & 33 & 104 & 0 & 0 & 11 & -0.0002 & $4.055 \times 10^{-5}$ & $<0.001$ \\
\hline Windspd $(\mathrm{m} / \mathrm{s})$ & 2.8 & 1.0 & 2.7 & 2.1 & 3.4 & -0.0187 & 0.0041 & $<0.001$ \\
\hline WindMax (m/s) & 5.2 & 1.2 & 5.0 & 4.3 & 5.8 & -0.0066 & 0.0032 & 0.0419 \\
\hline WindExt $(\mathrm{m} / \mathrm{s})$ & 8.6 & 2.2 & 8.2 & 7.0 & 9.8 & -0.0019 & 0.0017 & 0.278 \\
\hline Sunshine (h) & 4.7 & 4.0 & 4.8 & 0 & 8.2 & -0.0048 & 0.0010 & $<0.001$ \\
\hline
\end{tabular}

CVD deaths stands for the deaths for cerebrovascular diseases. PreAvg, PreMax, and PreMin stand for the daily average, maximum, and minimum atmospheric pressure, respectively. TemAvg, TemMax, and TemMin stand for the daily average, maximum, and minimum temperature, respectively. HumAvg and HumMin stand for the daily average and minimum humidity measured as percentage. RainVol stands for the daily rain volume. Windspd, WindMax, and WindExt are daily average, maximum, and extreme wind speed, respectively. SD stands for standard deviation. Q1 and Q3 stand for the first and third quartiles. $\beta$ stands for regression coefficient for the variable in generalized linear regression model, accompanied by SE (standard error) and $p$ value 
Table 2 Spearman correlation coefficients between the meteorological variables

\begin{tabular}{|c|c|c|c|c|c|c|c|c|c|c|c|c|c|}
\hline & PreAvg & PreMin & PreMax & TemAvg & TemMin & TemMax & HumAvg & HumMin & RainVol & Windspd & WindMax & WindExt & Sunshine \\
\hline $\mathrm{PM}_{2.5}$ & $0.24 *$ & $0.24 *$ & $0.23 *$ & $-0.33^{*}$ & $-0.37 *$ & $-0.26^{*}$ & $-0.08^{*}$ & $-0.23^{*}$ & $-0.15^{*}$ & $-0.34 *$ & $-0.21 *$ & $-0.22 *$ & -0.01 \\
\hline PreAvg & & $0.98^{*}$ & $0.98^{*}$ & $-0.87^{*}$ & $-0.87^{*}$ & $0.85^{*}$ & $-0.25^{*}$ & $-0.25^{*}$ & $-0.2^{*}$ & $-0.1^{*}$ & $-0.1^{*}$ & -0.04 & $-0.01 *$ \\
\hline PreMin & & & $0.96^{*}$ & $-0.85^{*}$ & $-0.84 *$ & $-0.82 *$ & $-0.26^{*}$ & $-0.25^{*}$ & $-0.2 *$ & $-0.1^{*}$ & $-0.16^{*}$ & $--0.09 *$ & 0.001 \\
\hline PreMax & & & & $-0.89 *$ & $-0.89 *$ & $-0.84 *$ & $-0.24 *$ & $-0.24 *$ & $-0.1^{*}$ & -0.08 & $-0.09 *$ & -0.01 & -0.04 \\
\hline TemAvg & & & & & $0.98 *$ & $0.98^{*}$ & $0.09 *$ & $0.12 *$ & 0.03 & $0.10 *$ & $0.09 *$ & 0.03 & $0.18 *$ \\
\hline TemMin & & & & & & $0.98 *$ & $0.18 *$ & $0.24 *$ & $0.11 *$ & $0.14 *$ & $0.09 *$ & 0.05 & 0.07 \\
\hline TemMax & & & & & & & 0.007 & $8 \times 10^{-4}$ & -0.04 & 0.07 & $0.09 *$ & 0.01 & $0.29 *$ \\
\hline HumAvg & & & & & & & & $0.90 *$ & $0.61 *$ & $-0.09 *$ & $-0.12 *$ & $-0.10 *$ & $-0.67 *$ \\
\hline HumMin & & & & & & & & & $0.59 *$ & 0.05 & -0.03 & -0.005 & $-0.72^{*}$ \\
\hline RainVol & & & & & & & & & & 0.06 & 0.10 & $0.14 *$ & $-0.61 *$ \\
\hline Windspd & & & & & & & & & & & $0.75^{*}$ & $0.71 *$ & 0.04 \\
\hline WindMax & & & & & & & & & & & & $0.90 *$ & $0.08 *$ \\
\hline WindExt & & & & & & & & & & & & & 0.02 \\
\hline
\end{tabular}

PresAvg, PreMax, and PreMin stand for the daily average, maximum, and minimum atmospheric pressure, respectively. TemAvg, TemMax, and TemMin stand for the daily average, maximum, and minimum temperature, respectively. Humvg and HumMin stand for the daily average and minimum humidity measured as a percentage. RainVol stands for the daily rain volume. Windspd, WindMax, and WindExt are daily average, maximum, and extreme wind speed, respectively. ${ }^{*} p<0.05$

$\mu \mathrm{g} / \mathrm{m}^{3}$ increment in $\mathrm{PM}_{2.5}$ concentration. All meteorological variables other than extreme wind speed showed a statistically significant association, and higher value tended to be associated with lower death risk apart from the daily atmospheric pressure variables.

There was multicollinearity among $\mathrm{PM}_{2.5}$ and meteorological variables (Table 2). Most of the correlation coefficients are statistically significant. For example, daily average atmospheric pressure has a highly negative relationship with the average temperature $(r=-0.87)$, average humidity is negatively correlated with the sunshine $(r=-0.67)$, and volume of rain is negatively correlated with the sunshine $(r=-0.61)$. According to the VIF values, daily average pressure, maximum pressure, minimum pressure, daily average temperature, maximum temperature, minimum temperature, daily average humidity, minimum humidity, maximum wind speed, and extreme wind speed are highly correlated to other variables and daily average rain volume, wind speed, and sunshine are moderately correlated to other variables (Table 3) (O'Brien 2007). For example, the VIF value of daily average pressure indicates that the variance of the estimated coefficient of the variable is inflated by a factor of 273.34 as it is highly correlated with at least one predictor variable. The correlations between $\mathrm{PM}_{2.5}$ and meteorological variables are rather weak, and most of them have a Spearman's $r<0.30$ (Table 3).

\section{Results of the GAM-PCA approach}

The first four principal components derived from PCA explained approximately $91 \%$ variation of the original data (Table 4). After adjusting for meteorological conditions

and day of the week, there was no longer a statistically significant association between daily $\mathrm{PM}_{2.5}$ concentration and CVD mortality in both $c r$ and $t p$ spline models. All the smoothing functions of the PCs are statistically significant except for the fourth PC.

Table 3 Variance inflation factor (VIF) of meteorological conditions

\begin{tabular}{ll}
\hline Variable & VIF \\
\hline PreAvg & 273.34 \\
PreMax & 97.96 \\
PreMin & 82.04 \\
TemAvg & 308.86 \\
TemMax & 118.51 \\
TemMin & 89.96 \\
HumAvg & 7.66 \\
HumMin & 10.97 \\
Rainvol & 1.30 \\
Windspd & 3.04 \\
WindMax & 6.52 \\
WindExt & 5.43 \\
Sunshine & 2.78 \\
\hline
\end{tabular}

PreAvg, PreMax, and PreMin stand for the daily average, maximum, and minimum atmospheric pressure, respectively. TemAvg, TemMax, and TemMin stand for the daily average, maximum, and minimum temperature, respectively. HumAvg and HumMin stand for the daily average and minimum humidity measured as a percentage. RainVol stands for the daily rain volume. Windspd, WindMax, and WindExt are daily average, maximum, and extreme wind speed, respectively 
Table 4 Summary of the GAM-PC analysis for the risk of cerebrovascular disease mortality

\begin{tabular}{|c|c|c|c|c|c|c|c|c|c|}
\hline & \multicolumn{2}{|c|}{ Parametric coefficients } & \multicolumn{3}{|l|}{ SE } & \multicolumn{4}{|l|}{$p$ value } \\
\hline & $c r$ & $t p$ & \multicolumn{2}{|l|}{$\mathrm{cr}$} & $t p$ & \multicolumn{2}{|l|}{$\mathrm{cr}$} & \multicolumn{2}{|l|}{$t p$} \\
\hline Intercept & 4.1 & 4.1 & \multicolumn{2}{|l|}{0.012} & 0.012 & \multicolumn{2}{|l|}{$<0.001$} & \multicolumn{2}{|l|}{$<0.001$} \\
\hline $\mathrm{PM}_{2.5}$ & $1.83 \times 10^{-5}$ & $3.97 \times 10^{-5}$ & \multicolumn{2}{|c|}{$1.15 \times 10^{-4}$} & $1.15 \times 10^{-4}$ & \multicolumn{2}{|l|}{0.87} & \multicolumn{2}{|l|}{0.73} \\
\hline DOW1 & $1.09 \times 10^{-2}$ & $1.08 \times 10^{-2}$ & \multicolumn{2}{|c|}{$1.45 \times 10^{-2}$} & $1.44 \times 10^{-2}$ & \multicolumn{2}{|l|}{0.45} & \multicolumn{2}{|l|}{0.45} \\
\hline DOW2 & $2.93 \times 10^{-3}$ & $2.42 \times 10^{-3}$ & \multicolumn{2}{|c|}{$1.44 \times 10^{-2}$} & $1.45 \times 10^{-2}$ & \multicolumn{2}{|l|}{0.84} & \multicolumn{2}{|l|}{0.87} \\
\hline DOW3 & $1.63 \times 10^{-2}$ & $1.58 \times 10^{-2}$ & \multicolumn{2}{|c|}{$1.44 \times 10^{-2}$} & $1.44 \times 10^{-2}$ & \multicolumn{2}{|l|}{0.26} & \multicolumn{2}{|l|}{0.27} \\
\hline DOW4 & $6.68 \times 10^{-3}$ & $6.55 \times 10^{-3}$ & \multicolumn{2}{|c|}{$1.44 \times 10^{-2}$} & $1.44 \times 10^{-2}$ & \multicolumn{2}{|l|}{0.64} & \multicolumn{2}{|l|}{0.65} \\
\hline DOW5 & $5.62 \times 10^{-3}$ & $5.53 \times 10^{-3}$ & \multicolumn{2}{|c|}{$1.44 \times 10^{-2}$} & $1.44 \times 10^{-2}$ & \multicolumn{2}{|l|}{0.70} & \multicolumn{2}{|l|}{0.70} \\
\hline DOW6 & $-1.31 \times 10^{-3}$ & $-1.31 \times 10^{-3}$ & \multicolumn{2}{|c|}{$1.44 \times 10^{-2}$} & $1.45 \times 10^{-2}$ & \multicolumn{2}{|l|}{0.93} & \multicolumn{2}{|l|}{0.92} \\
\hline \multicolumn{10}{|c|}{ The approximate significance of smoothing terms } \\
\hline & Edf & & \multicolumn{2}{|c|}{ Ref.df } & \multicolumn{2}{|l|}{ Chi-square } & \multicolumn{3}{|l|}{$p$ value } \\
\hline & $c r$ & $t p$ & $\mathrm{cr}$ & $t p$ & $c r$ & $t p$ & $c r$ & $t p$ & \\
\hline $\mathrm{s}(\mathrm{PC} 1)$ & 2.77 & 2.69 & 2.96 & 2.93 & 54.28 & 50.98 & $<0.001$ & $<0.001$ & \\
\hline $\mathrm{s}(\mathrm{PC} 2)$ & 1 & 1 & 1 & 1 & 7.22 & 6.04 & 0.007 & 0.01 & \\
\hline $\mathrm{s}(\mathrm{PC} 3)$ & 1 & 1 & 1 & 1 & 8.06 & 8.50 & 0.004 & 0.003 & \\
\hline $\mathrm{s}(\mathrm{PC} 4)$ & 1.55 & 1.44 & 1.83 & 1.74 & 1.40 & 0.50 & 0.43 & 0.63 & \\
\hline $\mathrm{s}(\mathrm{t})$ & 17.21 & 18.05 & 17.91 & 18.87 & 579.74 & 577.9 & $<0.001$ & $<0.001$ & \\
\hline$R$-sq.(adj) & & Deviance expla & & REML & & Scale e & & $N$ & \\
\hline $\mathrm{cr}$ & $t p$ & $\mathrm{cr}$ & $t p$ & $c r$ & $t p$ & $c r$ & $t p$ & $\mathrm{cr}$ & $t p$ \\
\hline 0.637 & 0.637 & $63.9 \%$ & $64 \%$ & 3990.6 & 3998.3 & 1 & 1 & 1091 & 1091 \\
\hline
\end{tabular}

$D O W$, the day of the week; $P C$, principal component; $S E$, standard error; $s($, smoothing function; $t$, time; $c r$, cubic regression; $t p$, thin plate; $E d f$, effective degrees of freedom; Ref.def, reference degrees of freedom; REML, restricted maximum likelihood; $N$, total number of observations. Parameter coefficients are adjusted estimates

\section{Results of the GAM shrinkage smoothers approach}

Both shrinkage smoothers ( $c r$ and $t p$ ) shrank daily average atmospheric pressure, maximum atmospheric pressure, minimum atmospheric pressure, minimum temperature, average humidity, average rain volume, average wind speed, maximum wind speed, extreme wind speed, and sunshine to zero (Table 5). Again, no statistically significant association was found between daily $\mathrm{PM}_{2.5}$ concentration and CVD mortality after adjusting for meteorological conditions and day of the week. The smoothing terms of average and maximum temperature and daily minimum humidity retained statistically significant.

\section{Results of the GAM-LASSO approach}

Daily average temperature and minimum temperature were selected using the LASSO procedure and included in the model (Table 6). The association between daily $\mathrm{PM}_{2.5}$ concentration and cerebrovascular mortality became no longer statistically significant after adjustment, but the smoothing term of average temperature retained statistically significant.

\section{Discussion}

In the current study, we evaluated the effect of daily $\mathrm{PM}_{2.5}$ concentrations on the CVD deaths in Shanghai, China, using GAM analysis with three different approaches for controlling for collinear confounding variables while simultaneously taking into account the linear and nonlinear relationships between meteorological confounding variables and the number of deaths. The daily average $\mathrm{PM}_{2.5}$ concentration level in our data was $55 \mu \mathrm{g} / \mathrm{m}^{3}$, and this was much higher than the recommended levels for $\mathrm{PM}_{2.5}$ yearly average below $10 \mu \mathrm{g} / \mathrm{m}^{3}$ or 25 $\mu \mathrm{g} / \mathrm{m}^{3}$ by the World Health Organization or the European Union, respectively (Thunis et al. 2017). There was a $1.7 \%$ elevated risk for CVD death per $10 \mu \mathrm{g} / \mathrm{m}^{3} \mathrm{PM}_{2.5}$ concentration in the unadjusted model, but after adjustment for meteorological variables and temporal trend, the exposure to $\mathrm{PM}_{2.5}$ was no longer associated. The results were consistent in the three modelling techniques we used.

A body of research has shown that an increase in $\mathrm{PM}_{2.5}$ was linked to an elevated risk of stroke (Franklin et al. 2008; Leiva et al. 2013; Lin et al. 2017; Lisabeth et al. 2008; Shah et al. 2015; Wellenius et al. 2012), ischemic heart disease (Pope et al. 2002), myocardial infarction (Peters et al. 2001), and cerebrovascular mortality (Gutierrez-Avila et al. 2018), and 
Table 5 Summary of the GAM with shrinkage smoothers analysis for the risk of cerebrovascular disease mortality

\begin{tabular}{|c|c|c|c|c|c|c|c|c|}
\hline & \multicolumn{3}{|c|}{ Parametric coefficients } & \multicolumn{3}{|l|}{ SE } & \multicolumn{2}{|l|}{$p$ value } \\
\hline & $c r$ & \multicolumn{2}{|l|}{$t p$} & \multicolumn{2}{|l|}{$\mathrm{cr}$} & $t p$ & $c r$ & $t p$ \\
\hline Intercept & 4.1 & \multicolumn{2}{|l|}{4.1} & \multicolumn{2}{|c|}{0.012} & 0.012 & $<0.001$ & $<0.001$ \\
\hline $\mathrm{PM}_{2.5}$ & $6.9 \times 10^{-5}$ & \multicolumn{2}{|l|}{$1.5 \times 10^{-4}$} & \multicolumn{2}{|c|}{$1.1 \times 10^{-4}$} & $1.2 \times 10^{-4}$ & 0.52 & 0.16 \\
\hline DOW1 & $1.0 \times 10^{-2}$ & \multicolumn{2}{|l|}{$9.2 \times 10^{-3}$} & \multicolumn{2}{|c|}{$1.44 \times 10^{-2}$} & $1.44 \times 10^{-2}$ & 0.47 & 0.52 \\
\hline DOW2 & $4.1 \times 10^{-3}$ & \multicolumn{2}{|l|}{$3.1 \times 10^{-3}$} & \multicolumn{2}{|c|}{$1.44 \times 10^{-2}$} & $1.44 \times 10^{-2}$ & 0.78 & 0.83 \\
\hline DOW3 & $1.9 \times 10^{-2}$ & \multicolumn{2}{|l|}{$1.7 \times 10^{-2}$} & \multicolumn{2}{|c|}{$1.44 \times 10^{-2}$} & $1.44 \times 10^{-2}$ & 0.20 & 0.23 \\
\hline DOW4 & $7.0 \times 10^{-3}$ & \multicolumn{2}{|l|}{$6.1 \times 10^{-3}$} & \multicolumn{2}{|c|}{$1.45 \times 10^{-2}$} & $1.44 \times 10^{-2}$ & 0.63 & 0.68 \\
\hline DOW5 & $5.6 \times 10^{-3}$ & \multicolumn{2}{|l|}{$4.5 \times 10^{-3}$} & \multicolumn{2}{|c|}{$1.44 \times 10^{-2}$} & $1.44 \times 10^{-2}$ & 0.70 & 0.75 \\
\hline DOW6 & $-1.4 \times 10^{-3}$ & \multicolumn{2}{|l|}{$-2.1 \times 10^{-3}$} & \multicolumn{2}{|c|}{$1.44 \times 10^{-2}$} & $1.44 \times 10^{-2}$ & 0.92 & 0.88 \\
\hline The approximate si & cance of smoot & terms & & & & & & \\
\hline & Edf & & Ref.df & & Chi-squ & & $p$ value & \\
\hline & $c r$ & $t p$ & $c r$ & $t p$ & $c r$ & $t p$ & $c r$ & $t p$ \\
\hline $\mathrm{s}(\operatorname{PreAvg})$ & $6.6 \times 10^{-4}$ & $2.3 \times 10^{-4}$ & 9 & 9 & 0 & 0 & 0.54 & 0.42 \\
\hline s(PreMax) & $1.3 \times 10^{-3}$ & 0.707 & 9 & 9 & 0.001 & 2.11 & 0.35 & 0.08 \\
\hline s(PreMin) & $6.9 \times 10^{-4}$ & $2.2 \times 10^{-4}$ & 9 & 9 & 0 & 0 & 0.68 & 0.85 \\
\hline s(TemAvg) & 2.48 & 4.40 & 9 & 9 & 8.07 & 49.83 & 0.005 & $<0.001$ \\
\hline $\mathrm{s}$ (TemMax) & 4.11 & 1.29 & 9 & 9 & 17.55 & 10.37 & $<0.001$ & $<0.001$ \\
\hline $\mathrm{s}(\mathrm{TemMin})$ & $6.8 \times 10^{-4}$ & $3.2 \times 10^{-4}$ & 9 & 9 & 0 & 0.001 & 0.73 & 0.38 \\
\hline s(HumAvg) & $5.2 \times 10^{-5}$ & 0.68 & 9 & 9 & 0 & 1.92 & 0.31 & 0.067 \\
\hline $\mathrm{s}($ HumMin) & 1.64 & 1.06 & 9 & 9 & 14.14 & 9.48 & $<0.001$ & $<0.001$ \\
\hline s(Rainvol) & $9.5 \times 10^{-4}$ & $2.5 \times 10^{-4}$ & 9 & 9 & 0 & 0 & 0.91 & 0.91 \\
\hline s(Windspd) & $4.4 \times 10^{-4}$ & $2.3 \times 10^{-4}$ & 9 & 9 & 0 & 0 & 0.63 & 0.87 \\
\hline s(WindMax) & $1.0 \times 10^{-3}$ & $6.5 \times 10^{-3}$ & 9 & 9 & 0.001 & 0 & 0.39 & 0.59 \\
\hline $\mathrm{s}($ WindExt $)$ & $4.4 \times 10^{-4}$ & $6.0 \times 10^{-4}$ & 9 & 9 & 0 & 0 & 0.84 & 0.75 \\
\hline $\mathrm{s}$ (Sunshine) & $5.1 \times 10^{-4}$ & $2.3 \times 10^{-4}$ & 9 & 9 & 0 & 0 & 0.82 & 0.82 \\
\hline $\mathrm{s}(\mathrm{t})$ & 8.94 & 8.94 & 9 & 9 & 369.12 & 329.99 & $<0.001$ & $<0.001$ \\
\hline$R$-sq.(adj) & Deviance exp & & REML & & & Scale estimate & $N$ & \\
\hline $\mathrm{cr}$ & $c r$ & $t p$ & $\mathrm{cr}$ & $t p$ & & $\mathrm{cr}$ & $c r$ & $t p$ \\
\hline 0.624 & $62.7 \%$ & $62.1 \%$ & 4000.7 & 401 & & 1 & 1091 & 1091 \\
\hline
\end{tabular}

$D O W$, the day of the week; $S E$, standard error; $c r$, cubic regression; $t p$, thin plate; $s()$, smoothing function; Edf, effective degrees of freedom; Ref.def, reference degrees of freedom; $R E M L$, restricted maximum likelihood; $N$, the total number of observations. Parameter coefficients are adjusted estimates

recent reviews showed that $\mathrm{PM}_{2.5}$ was associated with approximately $1 \%$ elevated risk of cerebrovascular mortality by every $10 \mu \mathrm{g} / \mathrm{m}^{3}$ increase (Shah et al. 2015; Wang et al. 2014). These studies revealed an increased risk of CVD associated with short-term exposure to $\mathrm{PM}_{2.5}$ in Europe, the USA, Asia, Africa, and South America. The excessive risk per $10 \mu \mathrm{g} / \mathrm{m}^{3}$ increase in $\mathrm{PM}_{2 \cdot 5}$ concentration ranged from $0.7 \%$ in mortality to $1.3 \%$ in hospital admission. In our data, the unadjusted association was similar but higher (1.7\% excessive mortality per $10 \mu \mathrm{g} / \mathrm{m}^{3}$ increase in $\mathrm{PM}_{2.5}$ concentration). It has been proved difficult to quantify premature mortality related to air pollution, notably in regions where air quality is not systematically monitored, and also because the toxic particles from various sources may vary (Tuomisto et al. 2008). The estimated effect of $\mathrm{PM}_{2.5}$ on premature mortality largely depends on the toxicity regarding the inhaled particle components. In China, emissions from residential energy use such as heating and cooking have the largest contribution to $\mathrm{PM}_{2.5}$, whereas in much of the USA and in a few other countries, emissions from traffic and power generation are important. In the eastern United States, Europe, Russia, and East Asia, agricultural emissions make the largest relative contribution to $\mathrm{PM}_{2.5}$ (Lelieveld et al. 2015). It might partially explain the difference in the findings between our study and other studies. On the other hand, the difference might be in part due to the profoundly different demographic characteristics, socioeconomic status, or environmental conditions. For example, Shanghai is a megacity with a dense population and high temperature and humidity all the year round, and $\mathrm{PM}_{2.5}$ may impact public health differently from other studied areas. Like the aforementioned studies, our study also controlled for the temporal trend of deaths and confounding from 
Table 6 Summary of the GAM analysis with selected variables by LASSO

\begin{tabular}{lllll}
\hline & Parametric coefficients & SE & $p$ value & \\
\hline Intercept & 4.11 & 0.012 & $<0.001$ & \\
PM $_{2.5}$ & $3 \times 10^{-5}$ & $1.1 \times 10^{-4}$ & 0.80 & \\
DOW1 & $9.8 \times 10^{-3}$ & 0.0144 & 0.50 & \\
DOW2 & $2.6 \times 10^{-3}$ & 0.0144 & 0.86 & \\
DOW3 & 0.017 & 0.0144 & 0.25 & \\
DOW4 & $4.6 \times 10^{-13}$ & 0.0145 & 0.75 & \\
DOW5 & 0.0041 & 0.0144 & 0.78 & \\
DOW6 & -0.0032 & 0.0145 & 0.82 & \\
The approximate significance of smoothing terms & & \\
& Edf & Ref.df & Chi-square & $p$ value \\
s(PressMax) & 2.39 & 3.09 & 3.98 & 0.26 \\
s(TemAvg) & 4.17 & 5.28 & 34.25 & $<0.001$ \\
s(TemMin) & 1.02 & 1.03 & 0.32 & 0.59 \\
s(t) & 17.18 & 17.89 & 464.62 & $<0.001$ \\
\hline
\end{tabular}

$D O W$, the day of the week; $S E$, standard error; $s()$, smoothing function; $E d f$, effective degrees of freedom; Ref.def, reference degrees of freedom

meteorological variables. However, we notice that when we adjusted for quite a few meteorological variables, the association became no longer statistically significant. The results were consistent across different modelling strategies, while the nonlinear association of CVD mortality with the meteorological variables retained. The observed $\mathrm{PM}_{2.5}$ concentrations in Shanghai were substantially higher than those in Europe or the USA where most of the current studies have been conducted and conclusions derived. The high ambient $\mathrm{PM}_{2.5}$ concentration level in itself might mask triggering effect of exposure, and the meteorological factors were only contributing to exacerbation of a $\mathrm{PM}_{2.5}$ exposure effect, which might be a potential reason that no statistically significant effect was observed for $\mathrm{PM}_{2.5}$ and deserves further investigation in the comparative studies using data from regions with low $\mathrm{PM}_{2.5}$ pollution..

The strengths of our study include, first, the adjustment using the rich information of meteorological variables enabled us for detailed control for weather conditions. Weather conditions and time-varying risk factors such as days of the week may cause a significant modification on $\mathrm{PM}_{2.5}$ levels and cerebrovascular events (Zhang et al. 2014). Second, multicollinearity among meteorological variables was handled using different approaches of PCA, shrinkage smoothers, and LASSO regularization, and the results were consistent in all the methods. Third, the use of two types of smoothing splines for the GAM model allowed us to compare results to minimize the bias from spline selection, and the results were, again, consistent regardless of types of smoothing splines. In the multicollinearity context, shrinkage methods, such as ridge regression, may reduce the dimensionality of the data by shrinking some coefficient estimates towards zero but not exactly to zero. While in LASSO, one of the correlated coefficients is usually zeroed and the other is assigned the entire impact. Because of this, ridge regression is expected to work better if there are many large parameters of about the same value. LASSO, on the other hand, is expected to come on top when only a few factors actually have impacts (James et al. 2013)

However, our study also has limitations. First, the relatively shorter time period for the analysis limited us to fully assess the long-term time trend in both $\mathrm{PM}_{2.5}$ pollution levels and cerebrovascular disease mortality. Second, only city-level $\mathrm{PM}_{2.5}$ concentrations from one air monitoring station were available in our study, but the concentration of $\mathrm{PM}_{2.5}$ may differ within the city and change during the day, and people's location would also not be constant. As a megacity with a population of about 24 million and an area of $6,340 \mathrm{~km}^{2}$, Shanghai has vastly different $\mathrm{PM}_{2.5}$ concentrations and meteorological conditions across the city. Although deaths from 16 administrative districts in Shanghai were available, only aggregated deaths of the whole city and the $\mathrm{PM}_{2.5}$ concentrations, as well as meteorological variables, from a single monitoring station were available in the study. It may mask and obscure the spatial and temporal variability of $\mathrm{PM}_{2.5}$ effects at particular exposure hotspots, such as the heat island effect in some parts of the city may exacerbate the effects of $\mathrm{PM}_{2.5}$ due to high temperatures that may also affect the outcomes. To overcome the lack of spatial variability in $\mathrm{PM}_{2.5}$ concentrations and/or meteorological data, a land use regression approach could be incorporated in the future (Liu et al. 2016). Of course, air pollution and mortality data from multisite would be more helpful for adjusting for the confounding from the spatiotemporal variability in $\mathrm{PM}_{2.5}$ concentrations and mortalities. Although many studies relied on air pollution information assuming a constant location of people at, for example, their residential addresses, the quantification of exposure using time and activity patterns of individuals will also enhance the understanding (Reis et al. 2018). Third, cerebrovascular mortality risk may vary by age, sex, and socioeconomic factors, but these characteristics were not controlled for in the current study. However, these characteristics tend to be stable within the city given our relatively short study period, thus confounding is unlikely (Pope et al. 2002). Fourth, we focused on the same day's effect of a single pollutant and did not include multiple pollutants (Wang et al. 2014) or lagged effects (GutierrezAvila et al. 2018). Although it is possible that air pollution may cause death after a certain period of time, a systematic review indicates that the risk appeared hardly different by the inclusion of lags (Shah et al. 2015). 


\section{Conclusion}

In this study, we aimed to evaluate the effect of $\mathrm{PM}_{2.5}$ pollution on CVD mortality using GAM with three different approaches for controlling for confounding factors. The initially statistically significant $1.65 \%$ elevated risk of CVD death was no longer observed after adjusting for a number of meteorological variables. As a large number of people are exposed to air pollution, further analysis using data with various measurement times, periods, and detailed pollutant and exposure profiles would contribute to enhancing the understanding of the impact of $\mathrm{PM}_{2.5}$ on human health and its mechanism.

Acknowledgments We thank Bo Fang, Chunfang Wang, and Tian Xia from the Shanghai Municipal Center for Disease Control and Prevention for their cooperation in data retrieving and cleaning.

Author contributions Conceptualization: Yang Cao, Khadija Akter Leepe, and Xin Fang. Funding acquisition: Yang Cao. Data curation: Xin Fang. Formal analysis: Khadija Akter Leepe and Yang Cao. Methodology: Yang Cao, Xin Fang, Mei Li, and Khadija Akter Leepe. Project administration: Yang Cao. Resources: Yang Cao. Supervision: Yang Cao. Validation: Yang Cao, Xin Fang, and Mei Li. Writingoriginal draft: Khadija Akter Leepe, and Yang Cao. Writing - review and editing: Ayako Hiyoshi, Xin Fang, Yang Cao, and Mei Li.

Funding information This work was financially supported by the Strategic Grant, Karolinska Institutet, Sweden (Project No. C62400032), the Junior Faculty Grant of the Institute of Environmental Medicine, Karolinska Institutet (Project No. C62412022), and the Joint China-Sweden Mobility Grant of the Swedish Foundation for International Cooperation Research and Education (Grant No. CH2015-6145).

\section{Compliance with ethical standards}

Ethics approval The study was approved by the Ethical Review Committee of the Shanghai Municipal Center for Disease Control and Prevention (SCDC), China (approval number: SCDC2016-08).

Competing interests The authors declare no that they have no competing interests.

Disclaimer The funding bodies were not involved in the design of the study, data collection and analysis, interpretation of results, and writing the manuscript.

Open Access This article is distributed under the terms of the Creative Commons Attribution 4.0 International License (http:// creativecommons.org/licenses/by/4.0/), which permits unrestricted use, distribution, and reproduction in any medium, provided you give appropriate credit to the original author(s) and the source, provide a link to the Creative Commons license, and indicate if changes were made.

\section{References}

Correia AW, Pope CA 3rd, Dockery DW, Wang Y, Ezzati M, Dominici F (2013) Effect of air pollution control on life expectancy in the United States: an analysis of 545 U.S. counties for the period from 2000 to
2007. Epidemiology 24(1):23-31. https://doi.org/10.1097/EDE. 0b013e3182770237

Crawley M (2013) The R book, 2nd edn. John Wiley \& Sons, West Sussex

Fang X, Fang B, Wang CF, Xia T, Bottai M, Fang F, Cao Y (2017) Relationship between fine particulate matter, weather condition and daily non-accidental mortality in Shanghai, China: a Bayesian approach. PLoS One 12(11):e0187933

Fiordelisi A, Piscitelli P, Trimarco B, Coscioni E, Iaccarino G, Sorriento D (2017) The mechanisms of air pollution and particulate matter in cardiovascular diseases. Heart Fail Rev 22(3):337-347. https://doi. org/10.1007/s10741-017-9606-7

Franklin M, Koutrakis P, Schwartz J (2008) The role of particle composition on the association between PM2.5 and mortality. Epidemiology 19(5):680-689. https://doi.org/10.1097/EDE. 0b013e3181812bb7

Friedman J, Hastie T, Tibshirani R (2010) Regularization paths for generalized linear models via coordinate descent. J Stat Softw 33(1):1-22

Gutierrez-Avila I, Rojas-Bracho L, Riojas-Rodriguez H, Kloog I, Just AC, Rothenberg SJ (2018) Cardiovascular and cerebrovascular mortality associated with acute exposure to PM2.5 in Mexico City. Stroke 49(7):1734-1736. https://doi.org/10.1161/Strokeaha.118. 021034

James G, Witten W, Hastie T, Tibshirani R (2013) An introduction to statistical learning, vol 112. S pringer, New York

Jimenez-Conde J, Ois A, Gomis M, Rodriguez-Campello A, CuadradoGodia E, Subirana I, Roquer J (2008) Weather as a trigger of stroke: daily meteorological factors and incidence of stroke subtypes. Cerebrovasc Dis 26(4):348-354. https://doi.org/10.1159/ 000151637

Leiva MA, Santibanez DA, Ibarra S, Matus P, Seguel R (2013) A fiveyear study of particulate matter (PM2.5) and cerebrovascular diseases. Environ Pollut 181:1-6. https://doi.org/10.1016/j.envpol. 2013.05.057

Lelieveld J, Evans JS, Fnais M, Giannadaki D, Pozzer A (2015) The contribution of outdoor air pollution sources to premature mortality on a global scale. Nature 525(7569):367-371. https://doi.org/10. 1038/nature15371

Lin HL, Guo YF, Di Q, Zheng Y, Kowal P, Xiao JP et al (2017) Ambient PM2.5 and stroke effect modifiers and population attributable risk in six low- and middle-income countries. Stroke 48(5):1191-1197. https://doi.org/10.1161/Strokeaha.116.015739

Lin YL, Zou JL, Yang W, Li CQ (2018) A review of recent advances in research on PM2.5 in China. Int J Environ Res Public Health 15(3):438

Lisabeth LD, Escobar JD, Dvonch JT, Sanchez BN, Majersik JJ, Brown DL et al (2008) Ambient air pollution and risk for ischemic stroke and transient ischemic attack. Ann Neurol 64(1):53-59. https://doi. org/10.1002/ana.21403

Liu C, Henderson BH, Wang DF, Yang XY, Peng ZR (2016) A land use regression application into assessing spatial variation of intra-urban fine particulate matter (PM2.5) and nitrogen dioxide (NO2) concentrations in City of Shanghai, China. Sci Total Environ 565:607-615. https://doi.org/10.1016/j.scitotenv.2016.03.189

Lu F, Xu D, Cheng Y, Dong S, Guo C, Jiang X, Zheng X (2015) Systematic review and meta-analysis of the adverse health effects of ambient PM2.5 and PM10 pollution in the Chinese population. Environ Res 136:196-204. https://doi.org/10.1016/j.envres.2014. 06.029

Marra G, Wood SN (2011) Practical variable selection for generalized additive models. Computational Statistics \& Data Analysis 55(7): 2372-2387. https://doi.org/10.1016/j.csda.2011.02.004

O'Brien RM (2007) A caution regarding rules of thumb for variance inflation factors. Qual Quant 41(5):673-690. https://doi.org/10. 1007/s11135-006-9018-6

Peters A, Dockery DW, Muller JE, Mittleman MA (2001) Increased particulate air pollution and the triggering of myocardial infarction. Circulation 103(23):2810-2815 
Pope CA 3rd, Burnett RT, Thun MJ, Calle EE, Krewski D, Ito K, Thurston GD (2002) Lung cancer, cardiopulmonary mortality, and long-term exposure to fine particulate air pollution. JAMA 287(9): $1132-1141$

Reis S, Liska T, Vieno M, Carvell EJ, Beck R, Clemens T et al (2018) The influence of residential and workday population mobility on exposure to air pollution in the UK. Environ Int 121:803-813. https://doi. org/10.1016/j.envint.2018.10.005

Shah ASV, Lee KK, McAllister DA, Hunter A, Nair H, Whiteley W et al (2015) Short term exposure to air pollution and stroke: systematic review and meta-analysis. Br Med J 350:h1295

Shanghai Statistics Bureau (2014) Shanghai Statistical Yearbook. Retrieved from http://www.stats-sh.gov.cn/html/sjfb/tjnj/

Thunis P, Degraeuwe B, Pisoni E, Trombetti M, Peduzzi E, Belis CA, ... Vignati E (2017) Urban PM2.5 atlas - air quality in European cities. Retrieved from Luxemboug:

Tibshirani R (1996) Regression shrinkage and selection via the Lasso. Journal of the Royal Statistical Society Series B-Methodological 58(1):267-288

Tuomisto JT, Wilson A, Evans JS, Tainio M (2008) Uncertainty in mortality response to airborne fine particulate matter: combining European air pollution experts. Reliab Eng Syst Saf 93(5):732744. https://doi.org/10.1016/j.ress.2007.03.002

Turner MC, Krewski D, Pope CA 3rd, Chen Y, Gapstur SM, Thun MJ (2011) Long-term ambient fine particulate matter air pollution and lung cancer in a large cohort of never-smokers. Am J Respir Crit Care Med 184(12):1374-1381. https://doi.org/10.1164/rccm. 201106-1011OC
Wang Y, Eliot MN, Wellenius GA (2014) Short-term changes in ambient particulate matter and risk of stroke: a systematic review and metaanalysis. J Am Heart Assoc 3(4):e000983

Wellenius GA, Burger MR, Coull BA, Schwartz J, Suh HH, Koutrakis P et al (2012) Ambient air pollution and the risk of acute ischemic stroke. Arch Intern Med 172(3):229-234. https://doi.org/10.1001/ archinternmed.2011.732

WHO (2016) Ambient air pollution: a global assessment of exposure and burden of disease. WHO Press, Geneva

Yang Y, Cao Y, Li WJ, Li RK, Wang M, Wu ZL, Xu Q (2015) Multi-site time series analysis of acute effects of multiple air pollutants on respiratory mortality: a population-based study in Beijing, China. Sci Total Environ 508:178-187. https://doi.org/10.1016/j.scitotenv. 2014.11.070

Zhang YS, Li SS, Pan XC, Tong SL, Jaakkola JJK, Gasparrini A et al (2014) The effects of ambient temperature on cerebrovascular mortality: an epidemiologic study in four climatic zones in China. Environ Health 13(1):24

Publisher's note Springer Nature remains neutral with regard to jurisdictional claims in published maps and institutional affiliations. 